\title{
Modifications physiques du milieu et conséquences sur le comportement hydrologique des cours d'eau de la Sierra Madre Occidentale (Mexique) \\ Physical modifications of the environment and consequences on the hydrological behaviour of rivers in the western Sierra Madre (Mexico)
}

\section{Viramontes et L. Descroix}

Volume 15, numéro 2, 2002

URI : https://id.erudit.org/iderudit/705466ar

DOI : https://doi.org/10.7202/705466ar

Aller au sommaire du numéro

\section{Éditeur(s)}

Université du Québec - INRS-Eau, Terre et Environnement (INRS-ETE)

\section{ISSN}

0992-7158 (imprimé)

1718-8598 (numérique)

Découvrir la revue

Citer cet article

Viramontes, D. \& Descroix, L. (2002). Modifications physiques du milieu et conséquences sur le comportement hydrologique des cours d'eau de la Sierra Madre Occidentale (Mexique). Revue des sciences de l'eau / Journal of Water Science, 15(2), 493-513. https://doi.org/10.7202/705466ar

\section{Résumé de l'article}

Des recherches ont été menées dans la Sierra Madre Occidentale du Mexique afin de déterminer l'impact des modifications physiques du milieu (surpâturage et déboisement) sur le fonctionnement hydrodynamique des bassins. L'étude des régimes hydrologiques des deux principaux cours d'eau (le río Ramos avec un bassin de $7.100 \mathrm{~km}^{2}$ et le Sextin de $4.700 \mathrm{~km}^{2}$ ) composant le haut Nazas montre une variation possible du régime hydrologique qui pourrait être liée aux dégradations de l'espace. Cette modification du régime n'est pas visible dans les tendances annuelles des pluies et des écoulements. De ce fait, d'autres indicateurs sont utilisés dans la recherche de tendances. En utilisant des algorithmes ordinaires et un modèle global simple (le modèle Nazas) nous testons et proposons ici trois indicateurs du régime hydrologique:

1. la comparaison entre les coefficients d'écoulement de base et de crue montre une possible diminution de l'écoulement de base;

2. Les temps de réponse des bassins montrent une probable diminution. Cela signifie que la transformation de la pluie en débit pourrait devenir de plus en plus rapide

3. le degré de participation de l'humidité préalable du milieu montre une possible diminution des temps de ressuyage du sol.

Si les résultats sont peu significatifs statistiquement dans cette zone, tous les indicateurs montrent une possible modification du régime hydrologique. L'enjeu est de taille ; la sierra approvisionne en eau plus du quart de la superficie du Mexique. Les modifications du régime hydrologique pourraient avoir des conséquences graves sur le développement. 


\title{
Modifications physiques du milieu et conséquences sur le comportement hydrologique des cours d'eau de la Sierra Madre occidentale (Mexique)
}

\author{
Physical modifications of the environment \\ and consequences on the hydrological behaviour \\ of rivers in the western Sierra Madre (Mexico)
}

\section{VIRAMONTES ${ }^{1}$, L. DESCROIX ${ }^{2}$}

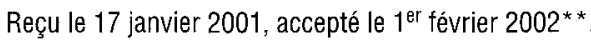

\section{SUMMARY}

Environmental impacts can alter the runoff regime of river basins. However, if the transformations are progressive, the hydrologic repercussions may not be always clearly visible. The influence that the physical parameters of the environment (soil surface features, vegetation, soil occupation, etc.) have on the hydrodynamic functioning of river basins is a current research concern in hydrological sciences. Generally these studies conclude that a decrease in vegetative cover leads to an increase in runoff, irrespective of the climate of the region.

For decades, the Western Sierra Madre has suffered progressive degradation by overgrazing and deforestation. This subtropical climate has a long dry season during the year ( 8 to 9 months), and the soils are generally thin (mostly Phaeozems and Lithosol). The vegetative cover of this zone is significantly degraded. Forests were reduced by more than $50 \%$ from 1972 to 1992 as a result of deforestation. Overgrazing also has impacted the landscape, leaving stony slopes and crusted surfaces observable all over the mountain.

Due to both overgrazing and deforestation, increased runoff coefficients and erosion rates have been observed at the plot and micro-plot scale in this region. The response is different at the basin scale, due to the spatial variability of the hydrodynamic behaviour of the slopes. The aim of this paper is to determine if over-exploitation of the terrestrial system could have hydrological consequences. New methods and indexes are proposed, which are able to highlight the hydrological responses of the basins. Examples are given for two watersheds of the Upper Nazas basin.

1. Instituto Mexicano de Tecnología del Agua (IMTA), Paseo Cuauhnáhuac 8532, CP 62550 Jiutepec, Morelos, México.

2. Laboratoire d'étude des transferts en hydrologie et l'environnement (LTHE), BP 53, 38041, Grenoble cedex 9, France.

* Correspondances. E-mail : davidv@tlaloc.imta.mx

** Les commentaires seront reçus jusqu'au 30 avril 2003. 
An examination of the two main rivers of the High Nazas (the Rio Ramos with a basin of $7100 \mathrm{~km}^{2}$ and the Sextin of $4700 \mathrm{~km}^{2}$ ) shows a likely variation of the hydrologic regime, which could be linked to spatial degradation. This modification of the regime is not visible in the annual trends of rain and runoff. As a result, ordinary algorithms and a simple global model (the Nazas model) were used to describe the annual trends. Three indicators of hydrologic regime are proposed here:

1. A comparison between the base-flow and flood-flow based on the algorithm of GUSTARD et al. (1989), which allows the separation of flood flow from base flow;

2. The lag time (or response time) of watersheds, the time necessary for the rain to cause an increase in river flow;

3. Changes in the water content of soil. Infiltration and evapo-transpiration rates are considered to be the main elements of water balance and it is generally assumed that they increase with increasing vegetation cover. This allows a greater volume of water to stay in the soil. When there is no soil water content assessment, the Antecedent Precipitation Index (API) can be used to compensate. This takes into account the volume of the previous rainfall events, the time passed since this event, and a parameter $\alpha$ of time water depletion.

Changes in land use were evaluated by remote sensing. The data collected were used in a calibration field campaign, to verify the hypothesis that there is a strong increase in overexploitation of the environment.

The results of the hydrological analysis were not always statistically significant in this zone. However, the changes are concomitant and all of the chosen indicators show a modification of the hydrologic regime. The analysis of $\mathbf{3 0}$ years of data shows that there is an evolution of the hydrologic behaviour of the watersheds. The following observations were made:

- a reduction of the base-flow was noticeable during the years 1970-1979 to 1990-1998. It appears that the floods have more and more importance in the hydrographs of the watersheds;

- a reduction in response time, which indicates that the transformation of the rainfall into runoff has become more and more rapid;

- the contribution of previous humidity in the environment in the formation of runoff also supports the earlier observations. It would seem that the drying time of the soil decreased. It appears to be a consequence of a reduction in soil water content, due to erosion and the degradation of vegetation.

The Western Sierra Madre supplies water to more than a quarter of the total area of Mexico. Changes in the hydrologic regime could have serious consequences on development in this region. The southern part of the country is mountainous, and $54 \%$ of the fields suitable for cultivation are located in the north of Mexico, but this region receives only $7 \%$ of the total water available in the whole country. The purpose of this research was to illustrate the importance of forests in the maintenance of the sustainability in the great "Laguna" irrigation district.

Key-words: watershed, base flow, lag time, soil water retention, overgrazing, deforestation, Northern Mexico.

\section{RÉSUMÉ}

Des recherches ont été menées dans la Sierra Madre occidentale du Mexique afin de déterminer l'impact des modifications physiques du milieu (surpâturage et déboisement) sur le fonctionnement hydrodynamique des bassins. 
L'étude des régimes hydrologiques des deux principaux cours d'eau (le río Ramos avec un bassin de $7100 \mathrm{~km}^{2}$ et le Sextin de $4700 \mathrm{~km}^{2}$ ) composant le haut Nazas montre une variation possible du régime hydrologique qui pourrait être liée aux dégradations de l'espace. Cette modification du régime n'est pas visible dans les tendances annuelles des pluies et des écoulements. De ce fait, d'autres indicateurs sont utilisés dans la recherche de tendances. En utilisant des algorithmes ordinaires et un modèle global simple (le modèle Nazas) nous testons et proposons ici trois indicateurs du régime hydrologique :

- la comparaison entre les coefficients d'écoulement de base et de crue montre une possible diminution de l'écoulement de base ;

- les temps de réponse des bassins montrent une probable diminution. Cela signifie que la transformation de la pluie en débit pourrait devenir de plus en plus rapide ;

- le degré de participation de l'humidité préalable du milieu montre une possible diminution des temps de ressuyage du sol.

Si les résultats sont peu significatifs statistiquement dans cette zone, tous les indicateurs montrent une possible modification du régime hydrologique. L'enjeu est de taille ; la sierra approvisionne en eau plus du quart de la superficie du Mexique. Les modifications du régime hydrologique pourraient avoir des conséquences graves sur le développement.

Mots clés : bassin versant, écoulement de base, temps de réponse, rétention en eau, surpâturage, déboisement, Nord-Mexique.

\section{1 - INTRODUCTION}

Les impacts des changements du milieu peuvent se traduire par des modifications du régime des écoulements. Cependant, si les transformations sont progressives dans le temps et diffuses dans l'espace (surpâturage, déforestation, urbanisation, etc.), les répercussions hydrologiques ne sont pas toujours perceptibles de manière très nette. Le rôle hydrologique des facteurs physiques du milieu (état de surface du sol, végétation, occupation du sol, etc.) est une des questions actuelles posées aux sciences de l'eau. Beaucoup d'aspects font l'objet d'études et parfois de controverses, notamment lorsqu'on évoque les conséquences des modifications du milieu sur les ressources en eau, les crues, les étiages, et l'érosion du sol.

\subsection{Grande variabilité de réponses}

L'influence de la couverture végétale et de ses modifications sur la partie terrestre du cycle de l'eau a été l'objet de nombreux travaux, qui montrent qu'en général, une diminution de la couverture végétale (déboisement, mise en culture, incendie, urbanisation, surpâturage) se traduit par une augmentation des écoulements. On peut citer en particulier, en climat tropical humide, les travaux de FRITSCH (1990), CALDER et al. (1995), woo et al. (1997) et SCOT et HUANG (1997) ; en climat méditerranéen, ceux de KOSMAS et al. (1997) et SORRiso et al. (1994); en climat tempéré, COSANDEY et al. (1990), GALEA et al. 
(1993), HUDSON et GILMAN (1993), et CROKE et al. (1998) ; en climat semi-aride et aride SNELDER et BRYAN (1995) et BERGKAMP (1998).

BOSCH et HEWLETT (1982), ont synthétisé les résultats des expérimentations sur 94 bassins versants de différentes régions du monde. Leurs résultats ont confirmé l'augmentation des coefficients d'écoulement avec le déboisement. Cependant, ils ont constaté que dans l'ensemble des résultats, la corrélation entre le taux de progression du boisement et la réduction de l'écoulement est médiocre. S'il est vrai que les écoulements augmentent après les coupes ou bien après les transformations de forêts en prairies ou en zones de culture, les résultats montrent une grande diversité d'explications qui évoquent la part de la végétation, des sols, et des modifications du milieu elles-mêmes (FRITSCH, 1990 ; COSANDEY, 1995 ; CAUGANT, 1998 ; ANDREASSIAN, 1999 ; COSANDEY et ROBINSON, 2000). Les seules données d'entrée (pluie) et de sortie (écoulement) de l'eau dans le bassin versant ne montrent pas la forte complexité du cheminement de l'eau parcouru dans le système. II a ainsi été démontré par STEDNICK (1996), à partir d'une compilation de données complétant celle de BOSCH et HEWLETT (1982), qu'il y a certes accroissement des coefficients d'écoulement avec le déboisement (mais la destruction de pâturages aurait un effet bien moindre), mais ce surtout à partir de $20 \%$ de la superficie du bassin déboisée ; cet auteur conclut aussi que « la variabilité des réponses de l'écoulement annuel à l'exploitation forestière suggère des processus complexes et non-linéaires ».

\subsection{Observations préalables à l'échelle expérimentale}

La région qui nous intéresse ici, la Sierra Madre occidentale, a subi une dégradation progressive (surpâturage et déforestation) de son manteau végétal et de ses sols depuis quelques décennies. Elle présente un climat subtropical avec une longue saison sèche dans l'année (8 à 9 mois), et des sols peu profonds (Phaeozems et Lithosols essentiellement). Les formations végétales dominantes sont une savane à chênes jusqu'à 2300 mètres d'altitude, une forêt de chênes et de pins au-dessus de cette limite. Or cette couverture végétale montre un état de dégradation alarmant. Les forêts ont été réduites de plus de $50 \%$ de 1972 à 1992 à cause du déboisement (RODRIGUEZ, 1997). De même, le surpâturage (la charge bétaillère est en moyenne trois fois supérieure à la capacité de charge des pâturages) a laissé des traces dans le paysage : des terrassettes, des versants caillouteux, des zones encroûtées, sont observables partout sur le terrain (BARRAL et ANAYA, 1995 ; POULENARD et al., 1996 ; VIRAMONTES, 1995).

Une augmentation des coefficients d'écoulement et des taux d'érosion liée à cette surexploitation du milieu (déboisement et surpâturage) a été mise en évidence par plusieurs travaux au niveau expérimental sur des parcelles de $1 \mathrm{~m}^{2}$ et $50 \mathrm{~m}^{2}$ (DESCROIX et NOUVELOT, 1997 ; VIRAMONTES, 2000). Cependant, ces effets locaux pourraient ne pas être observés à l'échelle régionale du fait de la grande variabilité spatiale et temporelle du milieu, déjà décrite dans cette zone (DESCROIX et al., 2001), et des précipitations (NOUVELOT et DESCROIX, 1996). La question qui se pose est de savoir si la surexploitation de l'espace peut avoir aussi des répercussions hydrologiques au niveau régional. Le but de cet article est également de décrire une méthode de recherche des indicateurs de tendance qui pourraient être plus sensibles que les valeurs totales ou 

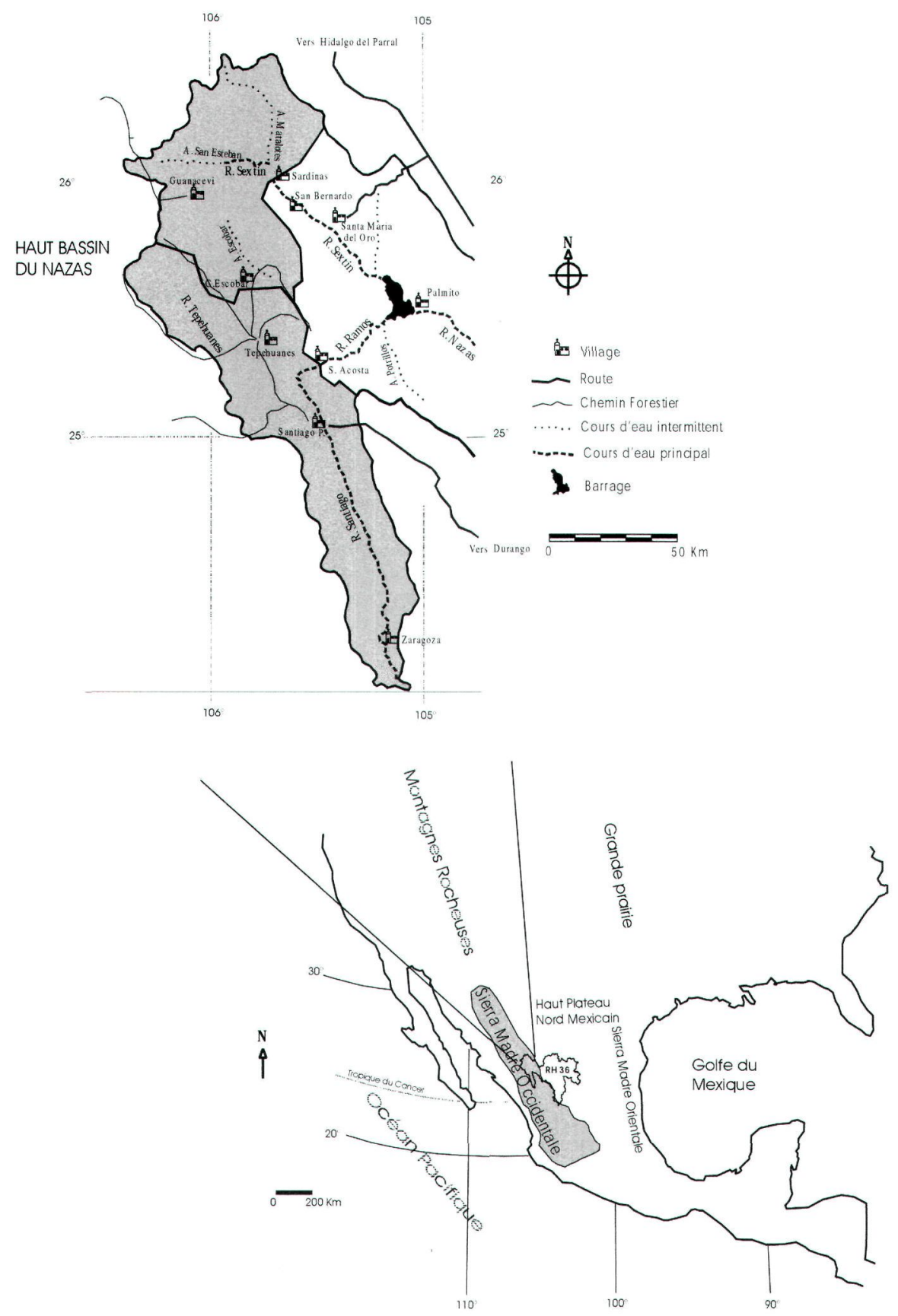

Figure 1 Localisation de la Sierra Madre occidentale et du haut bassin du Nazas.

Location of the Western Sierra Madre and the Upper Nazas basin. 
moyennes de débit. Ceci présente un intérêt tout particulier dans les cas où les volumes totaux annuels changent peu.

\section{2 - MATÉRIELS ET MÉTHODES}

Nous avons utilisé les données de deux grands bassins versants (figure 1) ayant des caractéristiques physiques et hydrologiques différentes (tableaux 1 et 2) mais connaissant la même surexploitation de l'espace (surpâturage et déforestation) : les bassins des ríos Ramos et Sextín, branches amont du río Nazas (état de Durango, Nord-Mexique). Trois types d'indicateurs basés sur le régime hydrologique sont testés ici :

- la comparaison des écoulements de base et de crue ;

- les temps de réponse des bassins versants ;

- l'importance de la capacité de stockage en eau des sols et des milieux. teurs.

Ci-après nous décrivons les méthodes utilisées pour obtenir ces indica-

\subsection{Comparaison des écoulements de base et de crue}

HUMBERT et KADEN (1994) ont proposé le rapport entre les écoulements de base et de crue (I'IEB, Indice d'écoulement de base) pour détecter d'éventuelles perturbations d'origine anthropique comme la construction des barrages dans les bassins versants. L'hypothèse de départ de cette comparaison est la suivante : "en admettant que les effets climatiques ont été éliminés, toute modification sensible des paramètres du milieu doit se répercuter sur l'évolution de la proportion respective du débit de base $(Q B)$ et du débit de crue (QC) ».

Dans un premier temps, une décomposition des hydrogrammes des deux cours d'eau (de 1970 à 1998 pour le Río Ramos et de 1971 à 1997 pour le Río Sextin) a été effectuée, le but étant d'analyser le rapport entre les écoulements de base et les écoulements de crue. Pour la séparation des hydrogrammes, on a utilisé le logiciel proposé par HUMBERT et KADEN (1994) basé sur la méthode de GUSTARD et al. (1989). Le principe de la décomposition de l'hydrogramme en écoulement de base et écoulement de crue est le suivant :

- à partir d'une chronique de débits journaliers, on divise la période de calcul en groupes de $N$ jours non chevauchés ( $N$ fixé à 3 dans notre cas) ; puis on recherche le minimum de chacun des groupes $(Q 1, Q 2, \ldots$ Qn) Le symbole $Q$ indique la valeur minimale d'un groupe de $N$ points donnés et l'indice " $n$ " réfère le nombre total de groupes de $N$ points non chevauchés;

- on scrute ensuite successivement les groupes (Q1, Q2, Q3), (Q2, Q3, Q4), $(Q i-1, Q i, Q i+1)$; si la valeur centrale d'un groupe Qi*PVM (PVM = pourcentage de validité des minima, fixé à 0,9 dans notre cas) est inférieure aux valeurs qui l'encadrent, celle-ci est alors retenue comme étant un point-pivot $(Q b 1)$. Dans ce cas $Q b i$ réfère le débit de base utilisé comme pivot pour la courbe du débit de base ; on répète l'opération $(Q b 2, Q b 3, \ldots$ 
Tableau 1 Caractéristiques physiques des bassins versants.

Table 1 Physical characteristics of the watersheds.

\begin{tabular}{|c|c|c|c|c|c|}
\hline Facteur & & \multicolumn{2}{|c|}{$\begin{array}{c}\text { Bassin du Río Ramos } \\
\left(7128 \mathrm{~km}^{2}\right)\end{array}$} & \multicolumn{2}{|c|}{$\begin{array}{l}\text { Bassin du Río Sextir } \\
\left(4660 \mathrm{~km}^{2}\right)\end{array}$} \\
\hline Pente & $\begin{array}{c}\text { Moyenne } \\
\text { Classe dominante }\end{array}$ & \multicolumn{2}{|c|}{$\begin{array}{c}17,0 \% \\
13 \text { à } 14 \%\end{array}$} & \multicolumn{2}{|c|}{$\begin{array}{c}19,9 \% \\
14 \text { à } 15 \%\end{array}$} \\
\hline Lithologie & $\begin{array}{l}\text { MEH } \\
\text { REM }\end{array}$ & \multicolumn{2}{|c|}{$\begin{array}{l}96,2 \% \\
3,8 \%\end{array}$} & \multicolumn{2}{|c|}{$\begin{array}{l}76,5 \% \\
23,5 \%\end{array}$} \\
\hline Sols & $\begin{array}{c}\text { Superficiels (0 à } 20 \mathrm{~cm} \text { ) } \\
\text { Moyens (20 à } 60 \mathrm{~cm} \text { ) } \\
\text { Profonds (plus de } 60 \mathrm{~cm} \text { ) }\end{array}$ & \multicolumn{2}{|c|}{$\begin{array}{l}28 \% \\
65 \% \\
7 \%\end{array}$} & \multicolumn{2}{|c|}{$\begin{array}{c}52 \% \\
45 \% \\
3 \% \\
\end{array}$} \\
\hline Végétation & $\begin{array}{c}\text { Année } \\
\text { Forêt } \\
\text { Prairies } \\
\text { Savane d'altitude } \\
\text { Buisson xérophile } \\
\text { Cultures }\end{array}$ & $\begin{array}{c}\mathbf{1 9 7 0} \\
77,7 \% \\
16,6 \% \\
4,3 \% \\
0,4 \% \\
1 \%\end{array}$ & $\begin{array}{c}1998 \\
42 \% \\
27 \% \\
29,5 \% \\
0,5 \% \\
1 \%\end{array}$ & $\begin{array}{c}1970 \\
76,7 \% \\
11,5 \% \\
10 \% \\
0 \\
1,8 \%\end{array}$ & $\begin{array}{c}1998 \\
48 \% \\
22 \% \\
28,5 \% \\
0 \\
1,5 \%\end{array}$ \\
\hline
\end{tabular}

MEH : Matériaux éruptifs hétérogènes (ignimbrites et tufs) ; REM : Matériaux consolidés massifs.

Tableau 2 Caractéristiques annuelles de pluie et d'écoulement des bassins du haut Nazas (bassin du Ramos, 1970 à 1998 et bassin du Sextin, 1971 à 1997).

Table 2 Annual rainfall and runoff characteristics of the upper Nazas watersheds (Ramos basin from 1970 to 1998 and Sextin basin from 1971 to 1997).

\begin{tabular}{cccccc}
\hline $\begin{array}{c}\text { Bassin } \\
\text { versant }\end{array}$ & Paramètre & $\begin{array}{c}\text { Lame } \\
\text { précipitée } \\
(\mathbf{m m})\end{array}$ & $\begin{array}{c}\text { Lame } \\
\text { écoulée } \\
(\mathbf{m m})\end{array}$ & $\begin{array}{c}\text { Coefficient } \\
\text { d'écoulement } \\
(\mathbf{\%})\end{array}$ & $\begin{array}{c}\text { Déficit } \\
\text { d'écoulement } \\
(\mathbf{m m})\end{array}$ \\
\hline \multirow{6}{*}{ Ramos } & Moyenne & $\mathbf{6 5 4 , 2 3}$ & $\mathbf{8 1 , 3 8}$ & $\mathbf{1 1 , 9 7}$ & $\mathbf{5 7 2 , 8 5}$ \\
& Écart-type & 111,64 & 46,20 & 5,55 & 84,88 \\
& Coeff. Variation & 0,17 & 0,57 & 0,46 & 0,15 \\
& Valeur Min. & 442,75 & 18,07 & 3,64 & 370,08 \\
& Valeur Max. & 886,66 & 213,05 & 24,03 & 730,41 \\
\hline \multirow{5}{*}{ Sextin } & Moyenne & $\mathbf{6 1 2 , 3 7}$ & $\mathbf{1 2 0 , 1 2}$ & $\mathbf{1 7 , 9 2}$ & $\mathbf{4 9 9 , 2 8}$ \\
& Écart-type & 121,83 & 90,84 & 11,00 & 76,03 \\
& Coeff. Variation & 0,20 & 0,76 & 0,61 & 0,15 \\
& Valeur Min. & 404,00 & 7,50 & 1,75 & 382,28 \\
& Valeur Max. & 871,19 & 419,86 & 51,20 & 646,59 \\
\hline
\end{tabular}

* Lames de pluie calculées à partir de 42 stations pluviométriques en mode krigeage.

Qbn). La valeur fixée pour PVM est arbitraire ; il s'agit de prendre une valeur proche de 1 et de ne pas changer cette valeur durant le traitement de toute la série; 
- par interpolation linéaire entre les Qbi, on estime chaque valeur de débit de base journalier $(Q b)$;

- enfin, on intègre les volumes annuels d'écoulement de base (VB), d'écoulement de crue $(V C)$, et d'écoulement total $(V T)$.

Dans notre cas, avec de grands écarts entre les données (de moins de $1 \mathrm{~m}^{3} \cdot \mathrm{sec}^{-1}$ à plus de $500 \mathrm{~m}^{3} \cdot \mathrm{sec}^{-1}$ ) et des variations très rapides de débits, nous avons fixé à 3 jours la valeur de « $N$ ", et à $90 \%$ la valeur de " $P V M$ ". De cette façon, nous avons un débit de base élevé, mais prenant plus en compte les variations des débits.

II s'agit d'une méthode linéaire classique basée sur les valeurs minimales de débit (figure 2). II faut donc accepter que la méthode de séparation des écoulements est précise mais basée sur des seuils prédéfinis, à ne pas modifier pour le traitement de tout l'échantillon. Cependant, avec un protocole rigoureux dans les deux séries de données (pour le río Ramos et le río Sextín) elle nous a permis de faire des comparaisons des résultats annuels obtenus.

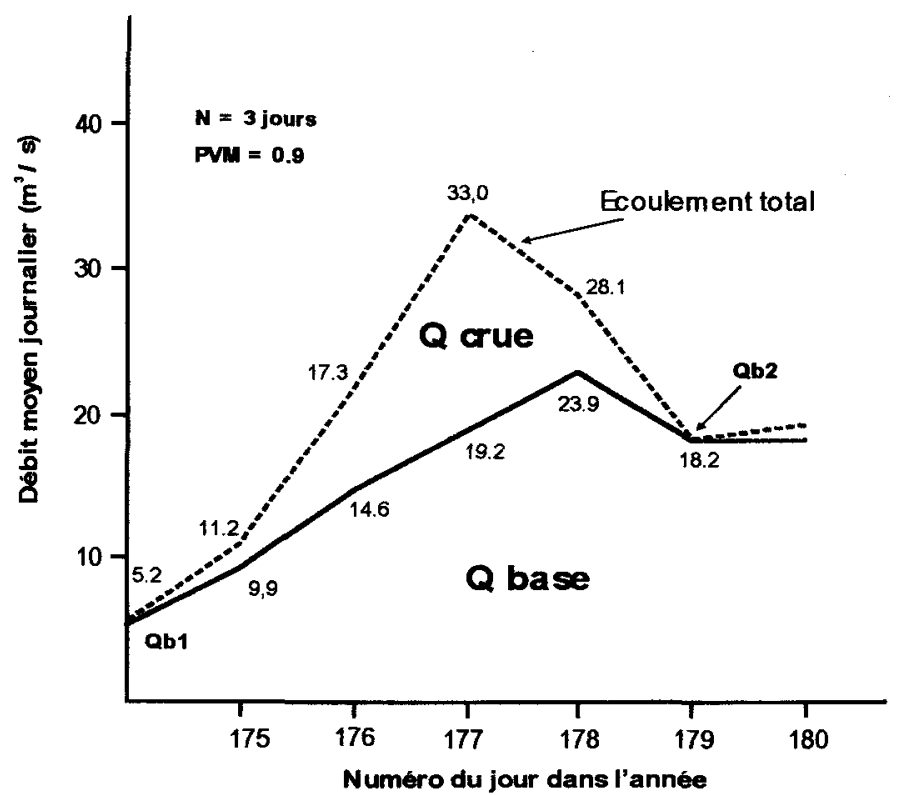

Figure 2 Exemple d'application de l'algorithme de GUSTARD et al. (1989) pour la séparation des hydrogrammes.

Example of application of the algorithm of GUSTARD et al. (1989) for the separation of hydrographs.

\subsection{Temps de réponse des bassins versants}

La méthodologie utilisée pour la mesure du temps de réponse dans les chroniques est relativement simple. Nous avons utilisé les données journalières de débits mesurés à l'exutoire des bassins versants et la chronique des pluies des stations météorologiques. Nous avons donc une mesure de pluie et de 
débit par jour seulement. Cependant, sur notre zone d'étude, les pluies se produisent une seule fois par jour dans plus de $90 \%$ des cas (NOUVELOT et DESCROIX, 1996), ce qui a facilité nos calculs. Une fois ordonnées les données journalières des pluies et des débits des deux bassins versants, nous avons utilisé un programme simple de calcul pour localiser la pluie et sa montée d'eau à l'exutoire du bassin :

- ordre des données journalières des stations pluviométriques $(P)$ et de débit de cours d'eau $(Q)$;

- localisation de l'augmentation de débit $(A)$ (différence entre $Q_{n}$ et $Q_{n-1}$ );

- localisation de la pluie correspondante à l'augmentation de débit. On cherche la pluie correspondante durant les trois jours précédant l'augmentation du débit : le jour même $\left(P_{n}\right)$, un jour avant l'augmentation de débit $\left(P_{n-1}\right)$ et 2 jours avant $\left(P_{n-2}\right)$;

- le temps de réponse est la différence en jours entre la pluie correspondante $\left(P_{n}\right)$ et l'augmentation de débit $(A)$. Si $C$ est présente en $P_{n-2},=2$ jours de temps de réponse (t.r.) ; $A$ en $P_{n-1}=1$ jour de t.r., et $A$ en $P_{n}=0,5$ jour de t.r.

Si la pluie s'étire sur plus d'un jour, il faudrait retirer les données de l'échantillon étudié.

Mise à part la faible résolution temporelle de la mesure, l'algorithme est bien sûr très rustique et présente quelques défauts. D'une part, les montées exceptionnelles des eaux peuvent masquer l'effet des petites pluies à l'exutoire du bassin versant. D'autre part, dans la période humide, il est fréquent que les pluies se présentent pendant plusieurs jours consécutifs. Auquel cas, l'algorithme estime toujours la moyenne des écarts entre les pluies et le début d'augmentation de débits. De plus, la distance entre les stations pluviométriques et l'exutoire du bassin versant n'est pas prise en compte mais l'on dispose de postes pluviométriques bien répartis dans le bassin. Le calcul de la lame précipitée s'effectue par krigeage à partir des postes dont les données sont disponibles pour l'événement.

Considérant toutes ces contraintes, il faut bien considérer que la mesure individuelle des événements est théoriquement précise au jour près alors qu'on décrit des variations de fractions de jours. Cela est rendu possible par le grand nombre d'évènements pris en compte (environ 2000 événements par bassin), par le calcul effectué toujours de la même manière et surtout, le fait qu'aucune tendance n'a été observée dans la série temporelle des pluies (NOUVELOT et DESCROIX, 1996 ; VIRAMONTES, 2000), qui viendrait entacher la représentativité d'une partie du jeu de données d'une erreur systématique. L'erreur de précision éventuelle est largement compensée par l'effet de masse d'une population nombreuse. Pour cette raison, nous avons utilisé toutes les chroniques journalières des pluies et des débits enregistrés dans les bassins versants du haut Nazas (de 1970 à 1998 pour le bassin du Ramos, et de 1971 à 1997 pour le bassin du Sextín).

\subsection{Importance de la capacité de stockage en eau des sols et des milieux}

L'humidité préalable des milieux (souvent ramenée à la teneur en eau du sol) revient souvent comme l'un des facteurs conditionnant le ruissellement dans les études sur parcelles et sur bassins versants expérimentaux (SITTNER 
et al., 1969 ; NOUVELOT, 1993 ; DE VRIES et HROMADKA, 1993 ; GRESILLON, 1994, entre autres) et aussi sur de grands bassins versants (ROSE, 1998). D'où l'utilisation fréquente d'un « Indice des précipitations antérieures » (API en sigle anglais) pour caractériser une teneur en eau théorique des sols à un moment précis (KOLHER et LINSLEY, 1951 ; CHEVALLIER, 1983 ; SEGUIS, 1987 ; NOUVELOT, 1993). Or, I'API simule relativement bien l'humidité du milieu sous des conditions hortoniennes (CASENAVE et VALENTIN, 1989) mais il peut apparemment aussi s'appliquer à certains bassins versants tempérés plus humides (SITTNER et al., 1969 ; ROSE, 1998).

On utilise ici un modèle hydrologique global simple : le modèle « Nazas». Ce modèle est basé sur l'hypothèse suivante (DESCROIX et NOUVELOT, 1997 ; VIRAMONTES et DESCROIX, 2001):

- la relation pluie-débit suivante est vérifiée pour tous les événements «pluie » $\mathrm{n}$ :

$$
\sqrt{R} d_{n}=K_{n}\left(P_{n}-P_{0 n}\right) \text { avec } P_{n}>P_{0 n}
$$

où $R_{n}$ et $P_{n}$ sont la lame écoulée et la précipitation respectivement, exprimées en $\mathrm{mm}$;

$\mathrm{K}_{\mathrm{n}}$ (en $\mathrm{mm}^{-1 / 2}$ ) est un paramètre qui dépend de la conductivité hydraulique de la surface du sol, de l'aire de l'impluvium concerné et de ses aires contributives à l'écoulement ;

$P_{0 n}(\mathrm{~mm})$ est la pluie limite d'imbibition (en deçà de laquelle il n'y a pas de ruissellement). Quand $P_{n}$ est significativement plus grand que $P_{0 n}$, il est facile de montrer que $K_{n}$ est relié à la variation du coefficient d'écoulement $\mathrm{RC}_{\mathrm{n}}$ (avec $\mathrm{RC}_{\mathrm{n}}=R \mathrm{~d}_{\mathrm{n}} / \mathrm{P}_{\mathrm{n}}$ ) avec le montant de la précipitation, par la relation : $\mathrm{K}_{\mathrm{n}}^{2} \approx \mathrm{dRC}_{\mathrm{n}} / \mathrm{dP}_{\mathrm{n}}$

- en assimilant le sol à un réservoir, $P_{0 n}$ peut être exprimé comme suit :

$$
P_{0 n}=C\left(H_{\max }-A P I_{n}\right) \text { avec } A P I_{n} \leq H_{\max }
$$

où $\mathrm{C}$ est un paramètre global qui tient compte de l'intensité de la pluie et de l'hétérogénéité de l'impluvium. $H_{\max }$ est la capacité maximale du réservoir $(\mathrm{mm})$ et $\mathrm{API}_{\mathrm{n}}(\mathrm{mm})$ son niveau à un moment donné.

- d'après la définition de l'Indice de précipitations antérieures (API), API est calculé ainsi :

$$
A P I_{n}=\left(A P I_{n-1}+P_{n-1}\right) \exp (-\alpha \Delta t)
$$

où $\Delta t=t_{n}-t_{n-1}$ est le temps (en jours et fraction) écoulé entre la fin de l'épisode pluvieux précédent $P_{n-1}$ est le début de l'épisode donné $\left(P_{n}\right)$. Le paramètre $\alpha$ (en jour ${ }^{-1}$ ) est l'inverse du temps de ressuyage du sol.

- en introduisant l'équation 3 dans l'équation 2 puis dans l'équation 1, on obtient :

$$
\sqrt{R} d_{n}=K_{n}\left\{P_{n}-C\left[H_{\max }-\left(A P I_{n-1}+P_{n-1}\right) \exp \left(-\alpha\left(t_{n}-t_{n-1}\right)\right]\right\}\right.
$$

L'équation (1) induit que chaque point de la relation $\sqrt{R} d_{n}\left(P_{n}\right)$ est inclus entre deux droites de pente $K_{\min }$ (pour une saturation minimale du sol) et $K_{\max }$ (pour une saturation maximale) qui croisent l'axe des abscisses en $P_{0 \max }$ et $\mathrm{P}_{\text {Omin }}$ respectivement. 
- de ce fait, $K_{n}$ peut être exprimé comme suit :

$$
K_{n}=K_{\min }+\left[\left(K_{\max }-K_{\min }\right) /\left(P_{\text {omax }}-P_{\text {omin }}\right)\right]^{*}\left(P_{\text {omax }}-P_{\text {on }}\right)
$$

où $\mathrm{K}_{\max }$ et $\mathrm{K}_{\min }$ sont respectivement les valeurs maximales et minimales $\mathrm{du}$ paramètre de la régression pour la parcelle ou le bassin concerné.

Le modèle (équation 4) a 7 paramètres $\left(C, H_{\max }, \alpha, K_{\max }, K_{\min }, P_{0 \max }, P_{\min }\right)$ qui peuvent être calibrés sur des séries de données observées.

Le modèle est initialisé au début de la saison des pluies quand $\mathrm{API}_{0}$ est supposé être égal à zéro. II est basé sur la supposition que l'écoulement est significativement influencé par l'humidité préalable du réservoir sol-végétation dans le bassin. II est assez classique de considérer que la prise en compte de l'Indice de précipitations antérieures améliore sensiblement la relation pluiedébit en conditions hortoniennes. Dans cet indice, le paramètre $\alpha$ représente la vitesse de décroissance de la teneur en eau du sol, et donc, sa capacité de rétention des eaux d'infiltration après un événement pluvieux.

Pour cette analyse, nous avons utilisé les données mensuelles des pluies et des écoulements des bassins versants du haut Nazas : de 1970 à 1998 pour le bassin du Ramos et de 1971 à 1997 pour celui du Sextín. Les lames mensuelles précipitées ont été calculées par interpolation en mode krigeage. Les lames écoulées mensuelles ont été calculées à partir des données journalières de débits et la superficie des bassins versants. Les données journalières d'écoulement correspondantes à chaque mois sont déphasées d'une journée de retard (pour tenir compte du temps de réponse du bassin).

\section{3 - RÉSULTATS}

Dans la recherche de tendances statistiques on a utilisé les tests proposés par le logiciel KHRONOSTAT de l'IRD (ORSTOM, 1988). Les résultats des tests statistiques vérifiant le caractère aléatoire : corrélation sur le rang (KENDALL et STUART, 1977) et autocorrélogramme (CHATFIELD, 1989) et la détection de ruptures : statistique U de BUISHAND (1984), ellipse de contrôle (BOIS, 1986), test de MANN-WHITNEY (PETTITT, 1979), méthode de segmentation des séries hydrométéorologiques (HUBERT et al., 1989) ; de deux bassins versants ne montrent pas de tendances définies à la hausse ou à la baisse de pluies et d'écoulements. Tous ces tests permettent de s'assurer qu'il n'y a pas de tendance dans les séries climatiques (VIRAMONTES, 2000).

Seule la méthode bayésienne de LEE et HEGHINIAN (1977) détecte des points de rupture dans les lames de pluie et d'écoulement et de coefficient d'écoulement (en 1993 pour le bassin Ramos et 1991 pour le bassin Sextin) . La figure 3 montre les résultats observés dans le bassin du Sextín. Cette rupture peut être expliquée en fonction de la variabilité des pluies des années 1990 avec une alternance d'années " humides " - à pluviométrie au-dessus de la moyenne (1991 et 1996) et d'années sèches (toutes les autres). En effet, contrairement à ce que l'on observe à l'échelle de la parcelle (augmentation des coefficients d'écoulement), la déforestation et le surpâturage de la Sierra Madre occidentale n'ont pas modifié les coefficients d'écoulement annuels des rivières. 


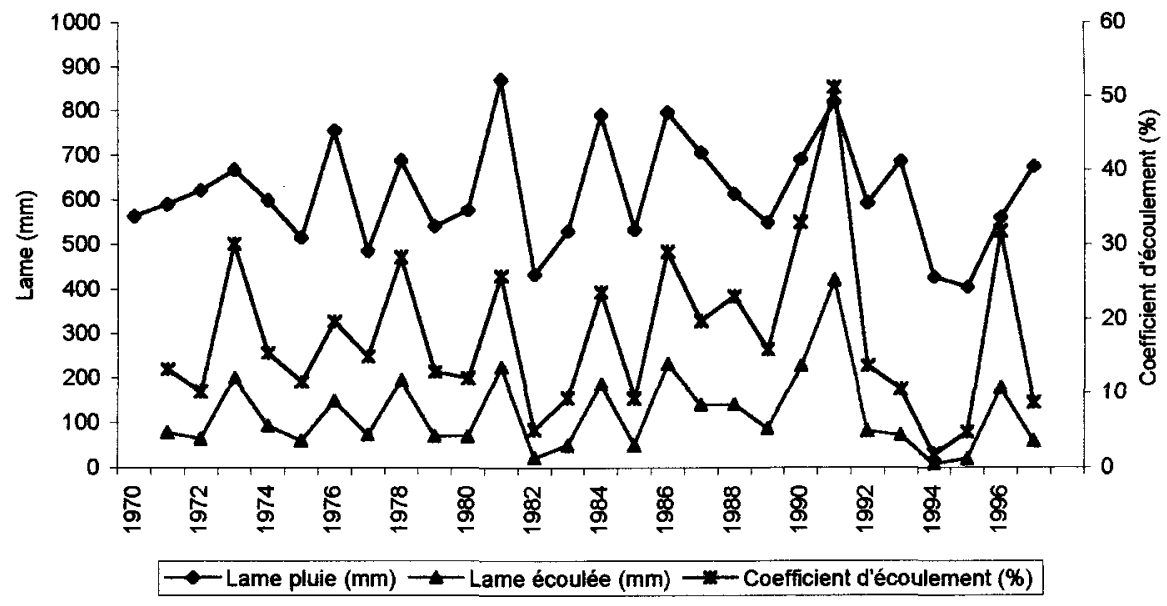

Test statistique

Hypothèse nulle (absence de rupture)

Acceptée

Rejetée

Caractère aléatoire (KENDALL et STUART, 1977 ;

CHATFIELD, 1989)

BUISHAND (1984) et ellipse de contrôle (BOIS, 1986)

MANN-WHITNEY (PETTITT, 1979)

LEE et HEGHINIAN (1977)

Segmentation HUBERT et al. (1989)

Figure 3 Valeurs annuelles de lames de pluie et d'écoulement ainsi que de coefficient d'écoulement du bassin du Sextin.

Annual values of runoff and rainfall depths, and the runoff coefficient for the Sextin basin.

Cependant, les chroniques analysées sont courtes (moins de 30 ans) et les conséquences hydriques de la transformation du milieu pourraient ne pas être sensibles dans les valeurs totales. Par la suite sont présentés les résultats des indicateurs basés sur le régime hydrologique.

\subsection{Concernant la comparaison des écoulements de base et de crue}

Dans les figures 4 et 5 , on présente les graphiques de régression linéaire entre les coefficients d'écoulement de crue et de base des cours d'eau, par périodes d'environ 15 années (la période totale d'observation a été divisée en deux), pour chacun des bassins versants. On a indiqué les droites de régression passant par l'origine. Les équations obtenues montrent des coefficients de détermination significatifs. Par ailleurs, ils sont représentatifs dans les deux cas suivant le test de Fischer-Snedecor. Par ailleurs, on constate que la pente de la droite de corrélation augmente d'une période à l'autre dans les deux bassins versants. Elle passe de 2,49 à 2,79 entre la période 1970-1984 pour le río Ramos et de manière plus tenue certes, pour le río Sextin pour les mêmes périodes (de 2,96 à 2,99). Cela témoigne d'une diminution de la part du volume 

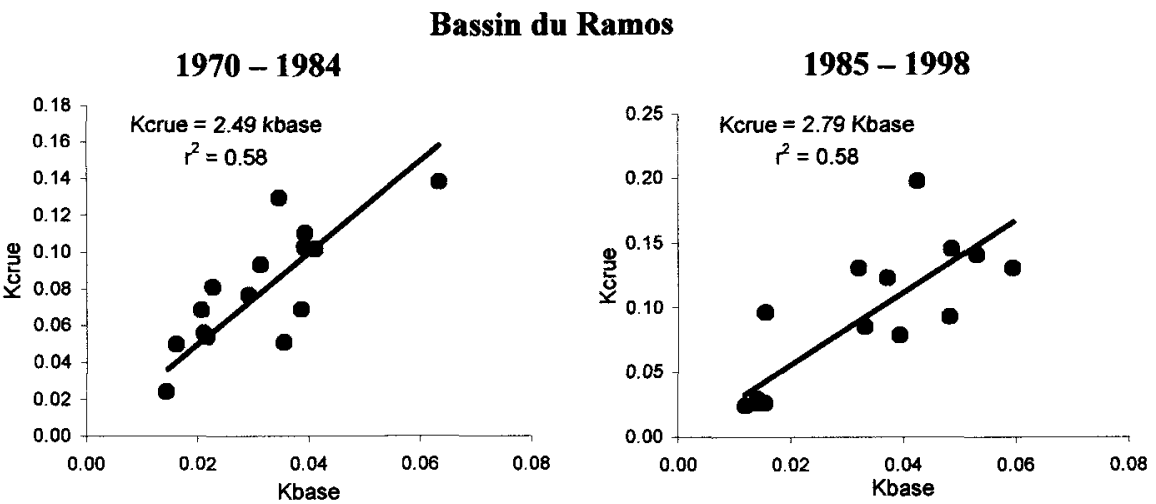

Figure 4 Évolution du rapport entre coefficient d'écoulement de crue (Kcrue) et coefficient d'écoulement de base (Kbase) pendant les périodes 19701984 et 1985-1998 dans le bassin du Ramos.

Evolution of the ratio Flood Runoff Coefficient (Kcrue)/Base Runoff Coefficient (Kbase) for the 1970-1984 and 1985-1998 periods in the Ramos basin.

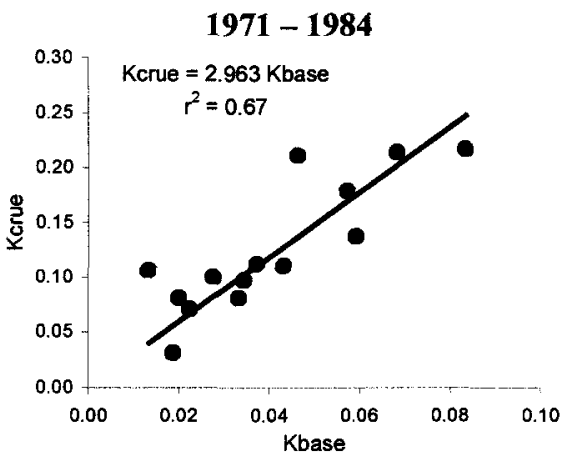

Bassin du Sextín

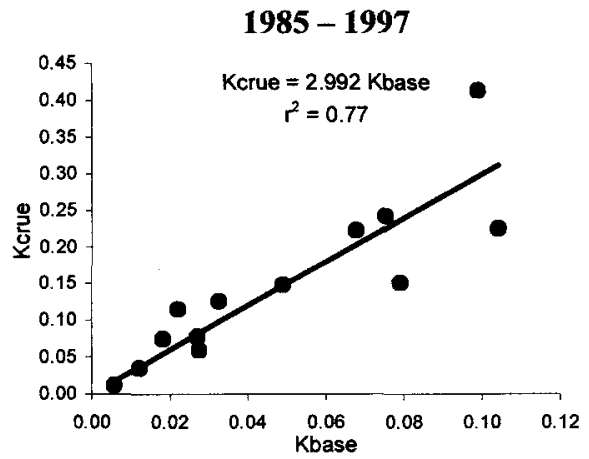

Figure 5 Évolution du rapport entre coefficient d'écoulement de crue (Kcrue) et coefficient d'écoulement de base (Kbase) pendant les périodes 19711984 et 1985-1997 dans le bassin du Sextin.

Evolution of the ratio Flood Runoff Coefficient (Kcrue)/Base Runoff Coefficient (Kbase) for the years 1970-1984 and 1985-1997 for the Sextin basin.

de base dans l'écoulement total annuel pour les deux bassins versants. Les écoulements de crue prennent donc de plus en plus de place dans l'écoulement total.

Cependant, il existe deux aspects statistiques qui empêchent une confirmation définitive de cette tendance : d'une part, le test de Student de comparaison des moyennes ne différencie (à $80 \%$ ) les deux groupes des valeurs de coefficient de base (Kbase) et de coefficient de crue (Kcrue), c'est-à-dire les deux périodes, que pour le bassin du Ramos. C'est-à-dire que les valeurs des deux 
périodes ont $80 \%$ de chances de provenir de populations différentes, mais uniquement pour ce bassin. La différence entre les deux périodes du bassin du Sextin n'est en revanche pas significative d'après ce test. Le deuxième facteur fait appel à la modération des résultats. En effet, il faut accepter que cette tendance est statistiquement faible du moment que nous avons des séries courtes (29 et 27 ans) comprenant des écarts très importants. Par ailleurs, l'augmentation de la pente de régression pour l'un des bassins (Sextin) est très faible.

\subsection{Concernant les temps de réponse des bassins versants}

Dans le tableau 3 sont présentés les résultats moyens de temps de réponse des deux bassins versants. En total, nous avons noté 2273 événements d'augmentation de débit dans le bassin du Ramos qui correspondent aux années 1970 à 1998, et 1720 événements pour le bassin du Sextín correspondant aux années 1971 à 1997. Les temps de réponse moyens des bassins est de 1,45 jours pour le bassin du Ramos, le plus grand, et de 1,32 jours pour le bassin du Sextín de taille sensiblement plus réduite. La distribution des résultats moyens par décennie montre une légère baisse dans le temps de réponse des deux bassins. Le bassin du Ramos présente une diminution de 1,6\%, mais cette diminution n'est pas significative d'après le test de comparaison de moyennes de Student (au seuil de $80 \%$ ). En revanche, dans le bassin du Sextín la moyenne passe de 1,32 jours dans les années 1970 à 1,26 jours dans les années 1990. La diminution des temps de réponse est de $5,5 \%$. Cette diminution est significative d'après le test de Student de différenciation des moyennes.

Tableau 3 Temps de réponse moyens total et par décennie des bassins versants.

Table 3 Mean response times of the watersheds for the total period and per decade.

\begin{tabular}{|c|c|c|c|c|c|c|}
\hline & & Total & $\begin{array}{r}\text { Sépa } \\
1970-1979\end{array}$ & $\begin{array}{c}\text { ation par déc } \\
1980-1989\end{array}$ & $\begin{array}{l}\text { ennie } \\
1990-1998\end{array}$ & $\begin{array}{c}\text { Différence } \\
\text { années } \\
1970 \text { et } 1990\end{array}$ \\
\hline \multirow[t]{6}{*}{$\begin{array}{l}\text { Bassin } \\
\text { Ramos }\end{array}$} & $\begin{array}{c}\text { Nombre } \\
\text { d'événements }\end{array}$ & 2273 & 836 & 847 & 590 & \multirow{5}{*}{$1,6 \%$} \\
\hline & $\begin{array}{l}\text { Moyenne } \\
\text { (jours) }\end{array}$ & 1,45 & 1,45 & 1,46 & 1,43 & \\
\hline & Écart-type & 0,38 & 0,38 & 0,36 & 0,40 & \\
\hline & $\mathrm{CV}$ & 0,26 & 0,26 & 0,25 & 0,28 & \\
\hline & \multirow[t]{2}{*}{ Student (80\%) } & & $*$ & $*$ & * & \\
\hline & & & 1971-1979 & $1980-1989$ & 1990-1997 & \\
\hline \multirow[t]{5}{*}{$\begin{array}{l}\text { Bassin } \\
\text { Sextin }\end{array}$} & $\begin{array}{c}\text { Nombre } \\
\text { d'événements }\end{array}$ & 1720 & 615 & 701 & 404 & \multirow{5}{*}{$5,5 \%$} \\
\hline & $\begin{array}{l}\text { Moyenne } \\
\text { (jours) }\end{array}$ & 1,32 & 1,34 & 1,34 & 1,26 & \\
\hline & Écart-type & 0,36 & 0,35 & 0,33 & 0,41 & \\
\hline & $\mathrm{CV}$ & 0,27 & 0,26 & 0,25 & 0,32 & \\
\hline & Student (80\%) & & $\checkmark$ & * & $\checkmark$ & \\
\hline
\end{tabular}

* Différence des moyennes non significative $; \checkmark$ Différence des moyennes significative. 
Par ailleurs, on observe certains points de rupture dans les chroniques du bassin du Sextín (tableau 4). L'hypothèse nulle (absence de rupture) est rejetée au seuil de confiance de $90 \%$ par les tests de BUISHAND (1984), de l'ellipse de contrôle (BOIS, 1986), de la méthode non paramétrique de PETITT (1979) et par la segmentation de HUBERT et al. (1989). Les points de rupture sont localisés en 1985 et 1996. Ces résultats confirment la diminution des temps de réponse du bassin du Sextín. Ainsi, le temps de réponse passe de 1,35 jours (entre 1971 et 1985) à 1,28 jours (entre la fin de 1985 et la fin de 1997).

Tableau 4 Résultats des tests statistiques du caractère aléatoire et de détection de rupture (seuil de confiance supérieur à $90 \%$ ) des temps de réponse des deux bassins versants.

Table 4 Results from statistical tests for the randomness and trends detection (at a 90\% confidence level) for the response times of the two basins.

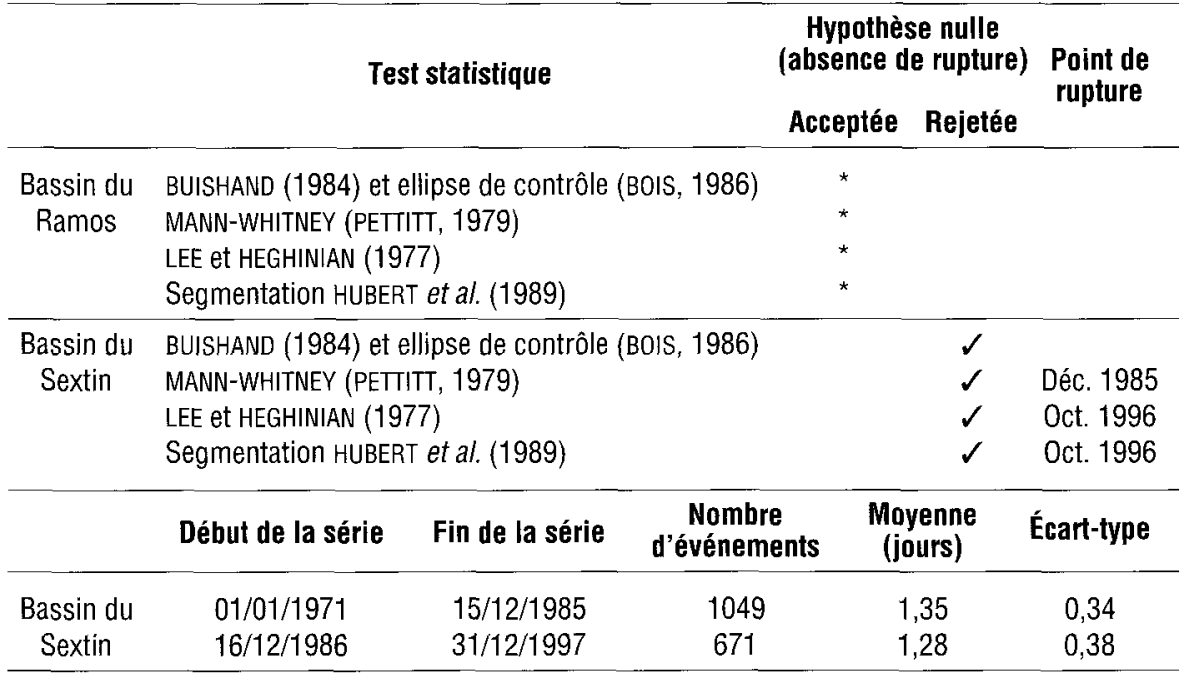

On a considéré ici non pas les crues, mais toutes les augmentations de débit ; c'est ce qui a permis d'avoir un échantillon important. On aurait pu se contenter des vraies crues (discriminées par un débit minimal ou par une augmentation de débit minimale) mais cela aurait réduit la taille de l'échantillon statistique.

\subsection{Concernant l'évolution du comportement de l'indice de précipitations antérieures à différentes périodes}

On a utilisé ici au pas de temps mensuel le modèle Nazas qui jusqu'à présent était utilisé en terme journalier. On présente d'abord la comparaison entre la lame écoulée observée et la lame écoulée calculée pour le modèle (figure 6). Les coefficients de détermination sont de 0,69 et 0,67 pour chacun des bassins. Donc la modélisation est satisfaisante. Les résultats obtenus par le modèle pour les séries mensuelles (totales et par décennie) de deux bassins versants se trouvent dans le tableau 5. Dans les deux bassins on remarque une 

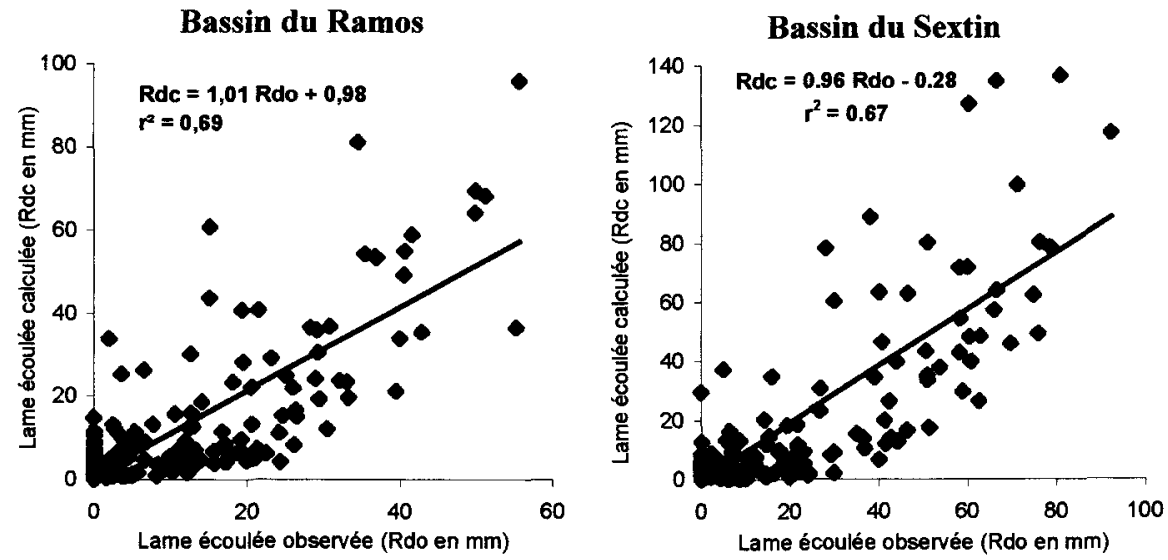

Figure 6 Comparaison entre la lame écoulée mensuelle observée $(R d o)$ et la lame écoulée mensuelle calculée $(R d c)$ par le modèle Nazas. Bassins versants des Ríos Ramos (1970-1998) et Sextin (1971-1997).

Comparison between monthly observed runoff depth (Rdo) and the monthly runoff depth calculated with the Nazas Model (Rdc). Ramos (1970-1998) and Sextin (1971-1997) basins.

Tableau 5 Relation entre la lame écoulée observée $(R d)$ (sans considérer l'API) et la lame écoulée calculée $(R d c)$ à partir du modèle Nazas (considérant l'API) et la précipitation $(P)$ dans les bassins du Ramos et du Sextin.

Table 5 Relationship between observed runoff depth $(R d)$ (without considering $A P I$ ), and runoff depth calculated with the Nazas model (considering $A P I)$, and rainfall $(P)$ on the Ramos and Sextin basins.

\begin{tabular}{|c|c|c|c|c|c|}
\hline \multirow{2}{*}{$\begin{array}{l}\text { Bassin } \\
\text { versant }\end{array}$} & \multirow{2}{*}{ Période } & \multicolumn{2}{|c|}{$\begin{array}{l}\text { Relation pluie-écoulement } \\
\text { sans considérer I'IPA }\end{array}$} & \multicolumn{2}{|c|}{$\begin{array}{l}\text { Relation pluie-écoulement } \\
\text { considérant I'IPA }\end{array}$} \\
\hline & & Équation & $r^{2}$ & Équation & $\mathbf{R}^{2}$ \\
\hline \multirow[t]{4}{*}{$\begin{array}{c}\text { Bassin } \\
\text { du Ramos }\end{array}$} & $\begin{array}{c}1970-1998 \\
n=348\end{array}$ & $R d=0,141 P$ & 0,492 & $R d c=0,137 P$ & 0,769 \\
\hline & $\begin{array}{c}1970-1979 \\
n=120\end{array}$ & $R d=0,135 P$ & 0,495 & $R d c=0,110 P$ & 0,748 \\
\hline & $\begin{array}{c}1980-1989 \\
n=120\end{array}$ & $R d=0,141 P$ & 0,505 & $R d c=0,139 P$ & 0,723 \\
\hline & $\begin{array}{c}1990-1998 \\
n=108\end{array}$ & $R d=0,147 P$ & 0,477 & $R d c=0,140 P$ & 0,775 \\
\hline \multirow[t]{4}{*}{$\begin{array}{l}\text { Bassin } \\
\text { du Sextin }\end{array}$} & $\begin{array}{c}1971-1997 \\
n=324\end{array}$ & $R d=0,238 P$ & 0,514 & $R d c=0,260 P$ & 0,860 \\
\hline & $\begin{array}{c}1970-1979 \\
n=108\end{array}$ & $R d=0,210 P$ & 0,504 & $R d c=0,237 P$ & 0,877 \\
\hline & $\begin{array}{c}1980-1989 \\
n=120\end{array}$ & $R d=0,229 P$ & 0,568 & $R d c=0,236 P$ & 0,868 \\
\hline & $\begin{array}{c}1990-1998 \\
n=96\end{array}$ & $R d=0,285 P$ & 0,508 & $R d c=0,287 P$ & 0,882 \\
\hline
\end{tabular}


amélioration du coefficient de détermination de la relation entre lame écoulée observée et lame précipitée en prenant en compte le modèle API (colonnes de droite) par rapport aux coefficients de régression sans prendre en compte l'humidité antérieure du milieu (colonnes de gauche). Dans les séries totales les coefficients de détermination de Rdc sont de 0,77 et 0,86 pour les bassins du Ramos et Sextin respectivement (contre 0,49 et 0,51 respectivement sans l'API). C'est-à-dire que si on considère l'Indice de précipitations antérieures du milieu, en plus de la précipitation $P$, on arrive à expliquer $77 \%$ et $86 \%$ de la variance de l'écoulement mensuel. Ceci tend à faire penser que l'état d'humectation initial du milieu est un paramètre important dans la production des écoulements des deux bassins. Les écoulements mensuels sont le reflet des pluies mensuelles et des effets des pluies des mois antérieurs.

Les résultats de paramètres obtenus par le modèle se trouvent dans les tableaux 6 et 7. Le paramètre $\alpha$ de décroissance de l'humidité traduit le temps nécessaire pour que le sol reprenne son état initial d'humidité. $\alpha$ est l'inverse du temps de ressuyage du sol : plus $\alpha$ est élevé, et plus le temps de ressuyage du sol est court. On observe dans les résultats globaux que le bassin du Sextín présente une valeur de 0,04 et le bassin du Ramos de 0,031. Cela signifie que le bassin du Ramos a un temps de ressuyage plus long que le bassin du Sextín. Ce qui coïncide avec les caractéristiques physiques et les valeurs de coefficient d'écoulement (le bassin versant de Sextín est plus « ruisselant " que celui du Ramos, car sa pente moyenne est plus forte et il est constitué de roches et sols moins perméables).

Tableau 6 Valeurs des paramètres du modèle Nazas par décennie du bassin du Río Ramos.

Table 6 Parameters values of the Nazas model per decade in the Ramos basin.

\begin{tabular}{lccccc}
\hline Paramètres du bassin du Ramos & \multicolumn{3}{c}{ Période } \\
& $\mathbf{1 9 7 0 - 1 9 9 8}$ & $\mathbf{1 9 7 0 - 1 9 7 9}$ & $\mathbf{1 9 8 0 - 1 9 8 9}$ & $\mathbf{1 9 9 0 - 1 9 9 8}$ \\
\hline Capacité maximale du réservoir $\left(H_{\max }\right)$ & 220 & 220 & 220 & 220 \\
Paramètre de décroissance de l'humidité $(\alpha)$ & $\mathbf{0 , 0 3 1}$ & $\mathbf{0 , 0 2}$ & $\mathbf{0 , 0 2 9}$ & $\mathbf{0 , 0 4}$ \\
\hline
\end{tabular}

Tableau 7 Valeurs des paramètres obtenus par le modèle Nazas par décennie du bassin du Río Sextin.

Table 7 Parameters values of the Nazas model per decade in the Sextin basin.

\begin{tabular}{lccccc}
\hline \multirow{2}{*}{ Paramètres du bassin du Sextin } & \multicolumn{4}{c}{ Période } \\
& $\mathbf{1 9 7 1 - 1 9 9 7}$ & $\mathbf{1 9 7 1 - 1 9 7 9}$ & $\mathbf{1 9 8 0 - 1 9 8 9}$ & $\mathbf{1 9 9 0 - 1 9 9 7}$ \\
\hline Capacité maximale du réservoir $\left(\mathrm{H}_{\text {max }}\right)$ & 130 & 130 & 130 & 130 \\
Paramètre de décroissance de l'humidité $(\alpha)$ & $\mathbf{0 , 0 4}$ & $\mathbf{0 , 0 4 0}$ & $\mathbf{0 , 0 5 4}$ & $\mathbf{0 , 0 6 1}$ \\
\hline
\end{tabular}

La comparaison du paramètre $\alpha$ par décennies nous montre la tendance suivante : sa valeur augmente, passant respectivement de 0,04 à 0,061 dans le 
bassin du Sextín et de 0,02 à 0,04 dans le bassin du Ramos entre les décennies 1970-1979 et 1990-1998. La tendance à la hausse du paramètre $\alpha$ indique que le temps nécessaire pour que le sol reprenne son état initial d'humidité a diminué. II semblerait que l'hypothèse initiale se confirme : le modèle utilisé permet de dire que le paramètre de décroissance de l'indice des précipitations antérieures (API) dans la production des écoulements, évolue dans le temps, donc que la capacité des bassins à stocker de l'eau diminue.

\section{4 - DISCUSSION}

En terme de volume total annuel, ni les pluies ni les écoulements des deux principaux cours d'eau du haut Nazas ne montrent de tendance définie à la hausse ou à la baisse. Apparemment, la déforestation et le surpâturage n'ont pas modifié les coefficients d'écoulement annuels des rivières de la Sierra Madre occidentale. Cela coïncide avec les résultats des travaux réalisé dans le Massif central par CAUGANT (1998) et ANDREASSIAN (1999) et dans les Appalaches (BENT, 1994). Ces auteurs n'ont pas constaté de relation entre les volumes annuels écoulés et la modification du couvert végétal. La détection difficile de tendances statistiques dans les chroniques dues aux séries trop courtes et aux erreurs de mesures supérieures aux tendances (problème évoqué par GALEA et BARBET, 1992), ainsi que la variabilité spatiale et temporelle du milieu, peuvent relativiser les effets de la transformation physique des bassins dans la production des écoulements. Ainsi, l'installation d'une végétation secondaire peut prendre rapidement la place de la végétation initiale (FRITSCH, 1990 ; BENT, 1994). STEDNICK (1996) insiste sur le fait que plus la pluviométrie moyenne est élevée, plus l'impact du déboisement est important, certes, mais il dure peu de temps, du fait que le temps de repousse de la végétation est très court.

De plus, les observations sur des stations expérimentales peuvent ne pas avoir de relation avec les résultats à une échelle supérieure à cause de la variabilité spatiale de l'infiltration (BERGKAMP, 1998 ; DESCROIX et al., 2001). Pour cette raison, la recherche des tendances hydriques ne doit pas se limiter aux séries de données totales de débits. Des paramètres de comportement du régime des bassins versants peuvent être des indicateurs plus sensibles aux changements ténus.

C'est l'ensemble des indicateurs qui nous permet de dire qu'il existe une modification possible du comportement hydrologique. En effet, le degré de signification statistique n'est pas toujours élevé pour chacun des indicateurs utilisés. II faut accepter que ces méthodes présentent certaines faiblesses : les algorithmes de la séparation des hydrogrammes et des temps de réponse sont de type arithmétique, c'est-à-dire que les résultats sont précis mais ils s'appuient sur des paramètres déterminés à l'avance et à ne pas modifier pendant le calcul de toute la série. De plus, la mise en évidence de la diminution du temps de ressuyage des bassins repose sur l'utilisation d'un modèle global simple. Cependant, nous avons respecté les protocoles de traitement des données, ce qui garantit une exactitude relative dans nos résultats. Or, sans pou- 
voir parler de preuves, on observe un faisceau de convergences. En effet, aucun des résultats obtenus n'est contradictoire. On peut constater un changement possible du comportement hydrologique de la zone qui se confirme dans l'ensemble des observations.

\section{5 - CONCLUSION}

Considérant que les changements climatiques des dernières 30 années ne sont pas significatifs (pas de tendance globale à l'augmentation ou à la baisse des précipitations ; VIRAMONTES, 2000), les activités humaines sont probablement les responsables de la modification possible du comportement hydrologique de la Sierra Madre occidentale.

Étant donnée l'importance du haut bassin du Nazas pour l'approvisionnement en eau de la région, cette modification possible des conditions du ruissellement peut entraîner de graves problèmes de développement. Au niveau local, il pourrait se traduire par une accentuation des crues et des étiages (les sols sèchent plus rapidement). Au niveau régional, le changement du régime hydrique pourrait se traduire par un accroissement des transports solides et une accélération du colmatage des barrages, ainsi qu'un accroissement de l'irrégularité interannuelle des écoulements. Or, le haut bassin du Nazas est représentatif d'une situation de surexploitation de toute la Sierra Madre occidentale. Elle représente le château d'eau des plus importants périmètres irrigués du Mexique. La modification observée des régimes hydriques peut provoquer des problèmes sévères de développement et de production agricole au niveau national. Ce risque pose au quotidien, surtout à l'heure actuelle dans les pays du Sud, le problème de l'adéquation de l'exploitation du milieu avec ses potentialités.

\section{RÉFÉRENCES BIBLIOGRAPHIQUES}

ANDRÉASSIAN V. (coordinateur), 1999. Indicateur d'impact de l'évolution du couvert forestier sur la ressource en eau à l'échelle des bassins versants des Cévennes et de la Montagne noire. Programme Environnement, Vie et Société CNRS, Rapport Final.

BARRAL H., ANAYA E., 1995. La ganadería y su manejo en relación con los recursos agua y pastizal en la zona semi-árida de México. Publicaciones ORSTOM-INIFAP. Gómez Palacio, México, 5, 78 p.

BENT G.C., 1994. Effects of timber cutting on runoff to Quabbin Reservoir, Central
Massachusetts. In: MARSTON R.A., HASFURTHER V.R. (eds), Effects of Human-Induced Changes on hydrologic Systems, AWRA Annual Summer Symposium. Am. Water Ress. Ass., 187196.

BERGKAMP G.E.R., 1998. A hierarchical view of the interactions of runoff and infiltration with vegetation and microtopography in semiarid shrublands. Catena, 33, 201-220.

BOIS P.H., 1986. Contrôle des séries chronologiques corrélées par étude du cumul des résidus. Deuxièmes journées hydro- 
logiques de l'Orstom. Montpellier, 89100.

BOSCH J.M., HEWLETT J.D., 1982. A review of catchment experiments to determine the effect of vegetation changes on water yield and evapotranspiration. Journal of Hydrology, 55, 3-23.

BUISHAND T.A., 1984. Tests for detecting a shift in the mean of hydrological time series. Journal of Hydrology, 58, 51-69.

CALDER I.R., HALL L.R., BASTABLE H.G., GUNSTON H.M., SHELA O., CHIRWA A., KAFUNDU R., 1995. The impact of land use change on the water resources in sub-Saharan Africa: a modelling study of lake malawi. Journal of Hydrology, 170, 123-135.

CASENAVE A., VALENTIN C., 1989. Les états de surface de la zone Sahélienne. Influence sur l'infiltration. Col. Didactique. Editions ORSTOM, Paris, 229 p.

CAUGANT C., 1998. Impact de l'évolution du couvert forestier sur le comportement hydrologique de bassins versants du Massif central. Mémoire DEA, Géomorphologie. Paris, Cemagref.

CHATFIELD C., 1989. The analysis of time series. An introduction. Fourth edition, Chapman and Hall, $241 \mathrm{p}$.

CHEVALLIER P., 1983. L'indice de précipitation antérieures. Évaluation de l'humectation des sols des bassins versants représentatifs. Cahiers ORSTOM, sér. Hydrolologie, XX (3-4), 179-189.

COSANDEY C., BOUDJEMLINE D., ROOSE E., LELONG F., 1990. Étude expérimentale du ruissellement sur des sols à végétation contrastée du Mont Lozère. Z., Géomorphologie, 34 (1), 6173.

COSANDEY C., 1995. La forêt réduit-elle l'écoulement annuel ? Annales de Géographie, 581-582, 7-25.

COSANDEY C., ROBINSON M., 2000. Hydrologie Continentale. Armand Colin éditeur, Paris, coll. " U », $360 \mathrm{p}$.

CROKE J., HAIRSINE P., FOGARTY P., 1999. Runoff génération and redistribution in logged eucalyptus forests, southeastern Australia. Journal of Hydrology, 216, 56-77.

DE VRIES J.J., HROMADKA T.V., 1993. Computer models for surface water. In: MAIDMENT, D.R. (Ed), Handbook of
Hydrology, chap. 21. McGraw-Hill, New York.

DESCROIX L., NOUVELOT J.F., 1997. Escurrimiento y erosión en la Sierra Madre Occidental. Publication ORSTOM-INIFAP, Gómez Palacio, Dgo. México. $50 \mathrm{p}$.

DESCROIX L., VIRAMONTES D., VAUCLIN M., GONZALEZ BARRIOS J.L., ESTEVES M., 2001. Influence of surface features and vegetation on runoff and soil erosion in the western Sierra Madre (Durango, North West Mexico). Catena, 43, 115-135.

FRITSCH J.M., 1990. Les effets du défrichement de la forêt amazonienne et la mise en culture sur l'hydrologie de petits bassins versants. Thèse Université de Montpellier II, $390 \mathrm{p}$.

GALEA G., BARBET D., 1992. Influence de la couverture végétale sur l'hydrologie des crues du BVRE du Mont Lozère. Hydrologie continentale, 7 (1), 33-49.

GALEA G., BREIL P., AHMAD A., 1993. Influence du couvert végétal sur l'hydrologie des crues, modélisation à validations multiples. Hydrologie continentale, $8(1), 17-33$.

GRESILLON J.M., 1994. Contribution à l'étude de la formation des écoulements de crue sur les petits bassins versants. Approches numériques et expérimentales à différentes échelles. Document d'Habilitation à Diriger des Recherches. UJF-LTHE, Grenoble, 158 p.

GUSTARD A., ROALD L.A., DEMUTH S., LUMADJENG H.S., GROSS R., 1989. Flow regimes from experimental and network data (FREND). Institute of Hydrology, Wallingford (UK), vol. 2.

HUBERT P., CARBONNEL J.P., CHAOUCHE A., 1989. Segmentation des séries hydrométéorologiques. Application à des séries de précipitations et de débits de l'Afrique de l'Ouest. Journal of Hydrology, 110, 349-367.

HUMBERT J., KADEN U., 1994. Détection des modifications de l'écoulement fluvial au moyen de l'indice de débit de base. Revue de Géographie Alpine, 82 (2), 2536.

HUDSON J.A., GILMAN K., 1993. Long term variability in the water balances of the Plynlimon cathments. Journal of Hydrology, 143, 355-380. 
KENDALL S.M., STUART A., 1977. The advanced theory of statistics. Charles Griffin London. 3, $585 \mathrm{p}$.

KOLHER M.A., LINSLEY R.K, 1951. Predicting the runoff from storm rainfall. Weather Bureau, U.S. Dep. of Commerce. Research Paper, $n^{\circ} 34$, Washington, $9 \mathrm{p}$.

KOSMAS C., DANALATOS N., CAMMERAT L.H., CHABART M., DIAMANTOPOULOS J., FARAND R., GUTIERREZ L., JACOB A., MARQUES H., MARTINEZFERNANDEZ J., MIZARA A., MOUSTAKAS N., NICOLAU J.M., OLIVEROS C., PINNA G., PUDDU R., PUIGDEFABREGAS J., ROXO M., SIMAO A., STAMOU G., TOMASI N., USAI D., VACCA A., 1997. The effects of land use on runoff and soil erosion rates under Mediterranean conditions. Catena, 29, 45-59.

LEE A.F.S., HEGHINIAN S.M., 1977. A shift of the mean level in a sequence of independent normal random variables, a Bayesian approach. Technometrics, 19 (4), 503-506.

NOUVELOT J.F., 1993. Guide des pratiques hydrologiques sur les petits bassins versants ruraux en Afrique tropicale et équatoriale. ORSTOM-CIEH, $539 \mathrm{p}$.

NOUVELOT J.F., DESCROIX L., 1996. Aridité et sécheresses du Nord-Mexique. Trace, CEMCA, México, 30, 9-25.

ORSTOM, 1998. Logiciel Khronostat, Projet FRIEND AOC.

PETTITT A.N., 1979. A non-parametric approach to the change-point problem. Applied Statistics, 28 (2), 126-135.

POULENARD J., DESCROIX L., JANEAU J.L., 1996. Surpâturage et formation de terrassettes sur les versants de la Sierra Madre occidentale (Nord-Ouest de Mexique). Revue de Géographie Alpine, Grenoble, 2, 77-86.

RODRIGUEZ M.G., 1997. Determinación de la vegetación en la Sierra Madre occidental para la calibración de imágenes satélite. ECF-UJED, Durango, México, $60 \mathrm{p}$.

ROSE S., 1998. A statistical method for evaluationg the effects of antecedent rainfall upon runoff: applications to the coastal plain of Georgia. Journal of Hydrology, 211, 168-177.

SCOTT MUNRO D., HUANG L.J., 1997. Rainfall, evaporation and runoff responses to hillslope aspects in the Shenchong Basin. Catena, 29, 131-144.

SEGUIS L., 1987. Indice des précipitations antérieures et prédiction des crues au Sahel. Hydrologie Continentale, 2 (1), 47-55.

SITTNER WT., SCHAUS C.E., MONRO J.C. 1969. Continuous Hydrograph Synthesis with an API-type Hydrological Model. Water resources research, 5 (5), 1007 1022.

SNELDER D.J., BRYAN R.B., 1995. The use of rainfall simulation tests to assess the influence of vegetation density on soil loss on degraded rangelands in the Baringo District, Kenya, Catena, 25, 105-116.

SORRISO-VALVO M., BRYAN R.B., YAIR A., LOVINO F., ANTRONICO L., 1994. Impact of afforetation on hydrological response and sediment production in a small Calambrian catchement. Catena, 25, 89-104.

STEDNICK J.D., 1996. Monitoring the effects of timber harvest on annual water yield. Journal of Hydrology, 176, 79-95.

VIRAMONTES P.D., 1995. Caracterización de los suelos y la vegetación en la parte alta de la cuenca del Nazas. Publicación ORSTOM-INIFAP, Gómez Palacio, Dgo. México. $45 \mathrm{p}$.

VIRAMONTES D., 2000. Comportement hydrodynamique du milieu dans le haut bassin du Nazas (Sierra Madre occidentale, Mexique). Causes et conséquences de son évolution. Thèse de l'université Joseph Fourier, Grenoble, 449 p.

VIRAMONTES D., DESCROIX L., 2001. Physical changes of environment and hydrologic consequences in the Western Sierra Madre (Mexico); experimental results and regional impacts. Communication, colloque «Michel Rieu », Paris, 8-10 octobre 2001.

WOO M.-K., FANG G., DICENZO P.D., 1997. The role of vegetation in the retardation of rill erosion. Catena, 29, 145-149. 